\title{
Analysis of an Axial T2 Weighted Brain MRI
}

\author{
Han Moi Sim1), Ishvi P.2)
}

\begin{abstract}
This paper simply encapsulate the recent developments in the field of Symmetry based approaches to detect tumor and then came up with a new parameter which plays a crucial role in proving that a high degree of Symmetry is present in an axial normal human brain MRI (Magnetic resonance Imaging) and this symmetry get affected or not present in anomalous cases highlighting the fact that tumor might be present. This paper tried to provide the proof of symmetry in human brain through a pixel based analysis using a number of neuro images cases. As Symmetry is one of the most significant property of a human brain that can be utilized to detect the presence of tumor and other anomalies present in the human Brain. For the pixel based analysis, a T2 weighted MRI modality images are used because in the T2 MRI images an anomaly appears Hyper intense (brighter than the normal brain tissue).
\end{abstract}

Keywords: symmetry, tumor; mean pixel value, T2 weighted, axial MRI, left hemisphere, right hemisphere.

\section{Introduction}

The numerous developments over the last decade or so in the field of computer technologies and Image segmentation are simply catalyzing the development of modern computerized approaches for anomaly detection in radiological images. However many major issues came into existence because computers generally lack sufficient perceptibility and intelligence in terms of discovering pathological patterns, which ultimately hinders the decision making process. As the anatomical knowledge plays a pivotal or indispensable role in computer vision and artificial intelligence [1-3], integrating anatomical knowledge into the computer system holds great promise for facilitating decision making and improving patient care. Based on the assumption that the brain exhibits a high level of bi-fold symmetry (Fig. 1) and that this symmetry is

Received(December 16, 2016), Review Result(1st: January 10, 2017, 2nd: February 24, 2017), Accepted(March 10, 2017)

${ }^{1}$ Department of Drone, Seoul Hoseo technical College 420, Gangseo-ro, Gangseo-gu, Seoul email: kmusic@naver.com

${ }^{2}$ (Corresponding Author) Department of Computer Science and Engineering, KL University email: ishvi12345@gmail.com 
violated in the presence of pathological anomalies conditions, many researchers have been motivated to construct a symmetry-based paradigm for automatic localization and segmentation of brain anomalies.

The general framework of this methodology is based on the hypothesis that the systematic correlation between asymmetry and pathologies can be a key to the improvement of existing detection algorithms. Integrating symmetry and asymmetry information as the prior knowledge or heuristics into a computer-aided diagnostic (CAD) [4-6] system, ought to enhance the system performance in the analysis of brain anomalies. To correctly quantify asymmetric patterns in brain images, however, the symmetry axis, or the symmetry plane, needs to be appropriately oriented in space. This enables the system to correct the possible misalignment of radiological scans and to evaluate hemisphere-wise asymmetry. Therefore, this paper has two main sections,

In the first section of this paper we tried to encapsulate the level of research already done in the field of Asymmetry and Symmetry based anomaly detection approaches while the second section contains a brief Pixel based symmetry analysis of a T2 MRI images highlighting or proving the fact that a high degree of symmetry is present in between the left and right hemisphere of a normal human brain, where as this symmetry is affected and not present in cases having tumor or any anomaly.
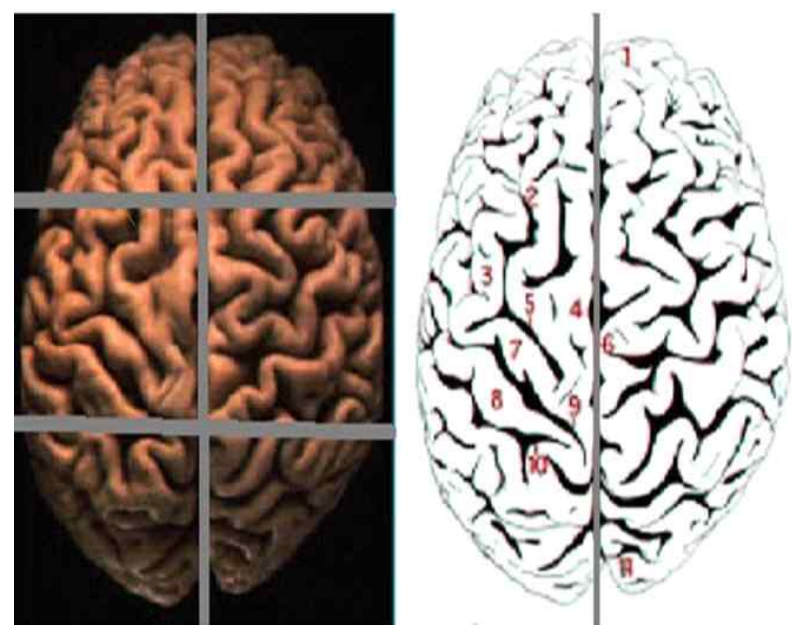

[Fig. 1] The brain torque demonstrates that the brain is largely symmetrical, but not perfectly symmetrical: The right frontal lobe (1) is larger than the left one, and the left occipital lobe is larger than the right one (11). This illustration is adapted from [7] 

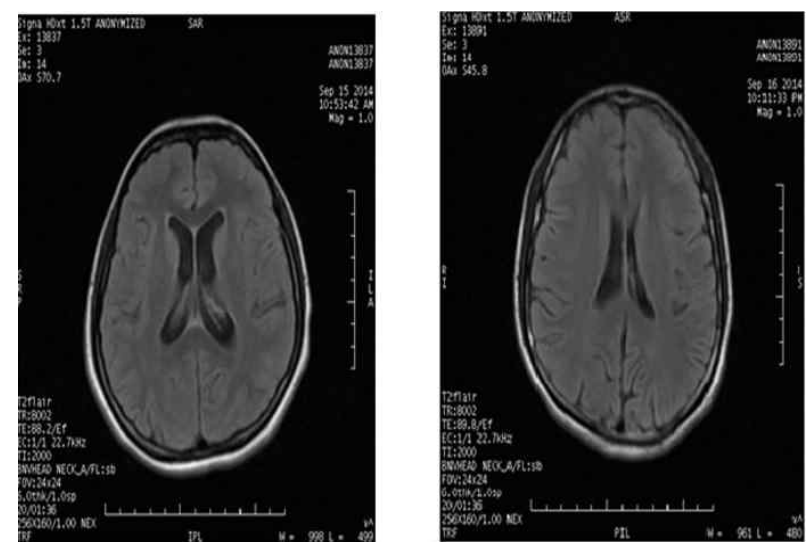

[Fig. 2] Normal Human Brain T2 Axial MRI scans

\section{Previous Works}

\subsection{The Meaning of Symmetry: From The Medical Perspective}

A normal human brain exhibits a extremely high level of bilateral symmetry, even though it is not perfectly symmetrical[8-12]. Corresponding regions of two hemispheres have approximately same anatomical properties, and also have comparable, if not identical, physiologic (e.g., blood perfusion) properties. The degree of asymmetry has long been thought to be helpful for suggesting a pathological condition and/or providing a diagnostic cue for clinicians. For example, abnormal asymmetry in the brain indicates a wide range of pathologies, such as stroke, bleeding and tumor. Radiologists routinely use symmetry/asymmetry as one of the most discriminating features, in conjunction with other characters such as location, neighborhood relationship, and shape, to assess abnormalities in brain images.

As per functionally wise, the brain is functionally asymmetrical and each side of the brain assumes distinctive functions. For instance, in most individuals, the left hemisphere is more active in linguistic tasks, while the right hemisphere is specialized for non-verbal tasks such as visual perception[9][13-16]. The degree to which anatomical asymmetry correlates with functional asymmetry remains questionable, but it is evident that morphological differences between hemispheres, however slight, occur systematically in normal brains. One example would be that the right frontal lobe is expected to be bigger than its left counterpart[9][12-21]. In any case, however, one has to be aware of the existence of normal asymmetry might potentially confound the process of extracting abnormal asymmetry. Both the Symmetry as 
well as little Asymmetry phenomenon of human brain can be used to detect the tumor and other anomalies.

\subsection{Research in the Field of Symmetry based Approaches to Detect Brain Tumor}

The property of Symmetry is a well known means to discover the structure of objects and it was used in many domains. Most of the approaches based on using a priori knowledge on the image and the kind of symmetry that we are looking for (rotational or bilateral). The very first study was made by Atallah [22] and it required objects to be presented as points, lines or circles. Some morphological methods as thinning or "grass fire" were tested but only on a set of binary images. Xia [23] comes up with a good review paper, which also showcased the sensitivity of these methods. The first symmetry based detection approach which eventually does not need object recognition or segmentation was proposed by Reisfeld [24]. Then Kovesi [25] managed to compute symmetry without any a priori on the image by using the local phase. Keller [26] proposed an algebraic approach based on the Fourrier transform. In the medical imaging field symmetry was mainly applied to the brain were statistical measures and symmetry axis computation methods were proposed by Tuzikov [27]. A tumor detection method using histograms was first proposed by Wang [28].

In the year 2007, Nilanjan Ray, Baidya Nath Saha, and Matthew Robert Graham Brown proposed a technique for computing bounding boxes around brain abnormality in standard MR images based on symmetry. The technique uses a scoring function that provides a measure of the similarity or difference between two regions in terms of the Bhattacharya coefficient computed on those region intensity histograms. We provide a mathematical basis of the behaviour of this scoring function that essentially locates the bounding box. This region-based and image feature histogram-based approach can open new avenues of brain tumor boundary delineation [29].

In 2008, Nilanjan Ray, Russell Greiner and Albert Murtha come up with another technique based on Symmetry to detect anomaly in brain MRI[30]. In this approach they have simply proposed a real-time algorithm to locate the brain abnormality in an MR image by putting a bounding box around it. Their approach is based on left-to right symmetry of the brain. In additional to being real-time, some advantages of the proposed algorithm are, It requires no registration of MR images, It needs no training image and It is independent of intensity variations across MR images. Their detection algorithm can play a useful role in indexing and storage of MRI data and as an initial step toward accurate tumor boundary delineation. 
In the year 2010, Soniya Goyal [31] of IIT Delhi proposed a simple method based on symmetry to detect the brain tumor in the axial brain MRI. The approach simply based on the portioning of patient axial MRI image in module of fixed size and numbered them symmetrically and the compare these symmetrical modules present in left as well as in the right hemisphere. Although the approach was very simple but effective. She later on tests this approach on a number of test cases and proved to be very reliable.

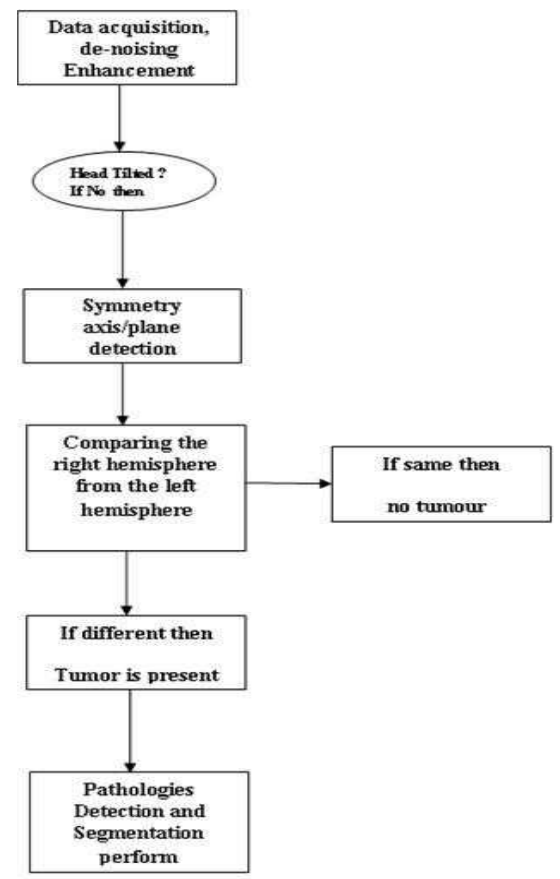

[Fig. 3] A general Flow diagram depicting the primary stages involve in a general symmetry based approach.

The next section of this paper simply contains the pixel intensities values based analysis of MRI images showcasing the presence of high degree of symmetry in normal patient MRI and the cases in which symmetry property totally got compromised and unable to detect tumor[32][33].

The T2 Axial MRI image is decomposed into eight modules or sections. Since the MRI images of $512 * 512$ standard sizes so each constituent module is of size $128^{*} 256$. The median pixel value gradient of each module of T2 MRI image can be calculated as: 


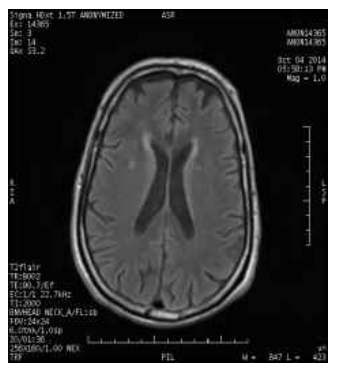

\begin{tabular}{|l|l|l|l|}
\hline $\mathrm{P} 1 * 1$ & $\mathrm{P} 1 * 2$ & - & $\mathrm{P} 1 * 256$ \\
\hline $\mathrm{P} 2 * 1$ & $\mathrm{P} 2 * 2$ & - & $\mathrm{P} 2 * 256$ \\
\hline- & - & - & \\
\hline $\mathrm{P} 128 * 1$ & $\mathrm{P} 128 * 2$ & - & $\mathrm{P} 128 * 256$ \\
\hline
\end{tabular}

[Fig. 4] Pixel Values based Symmetry Analysis of an Axial T2 Weighted Human Brain MRI

This pixel values based analysis is consist of three phases, the first phase is all about showcasing the symmetry quotient present in the Axial human brain MRI with the help of a new parameter as mean pixel value gradient. The mean pixel value gradient can be simply calculated with help of a Matlab code that first convert a T2 MRI scan present in DICOM format (It is the standard used worldwide for the transmission of Medical images) into $512 * 512$ standard grey level image.

Then the Matlab code simply partition this standard size grey level image into eight constituent modules or sections. The four modules on the left simply represent the left hemisphere and the remaining four modules on the right represent the right hemisphere. The matlab code now computes the mean pixel values of each module or section which is presented below:

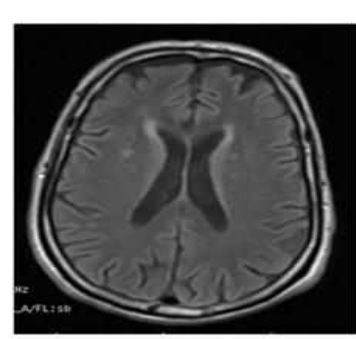

\begin{tabular}{|l|l|}
\hline 20.0742 & 21 \\
\hline 44.31 & 45.20 \\
\hline 51.02 & 51.90 \\
\hline 36.8410 & 35.02 \\
\hline
\end{tabular}

[Fig. 5] T2 MRI scan of a normal brain and its corresponding mean pixel value table

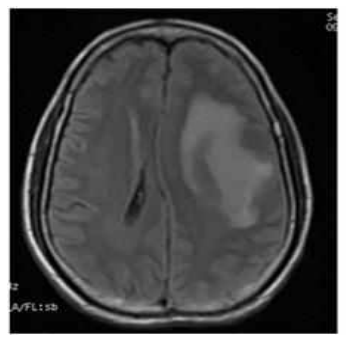

\begin{tabular}{|l|l|}
\hline 19.06 & 20.01 \\
\hline 36.77 & 44.52 \\
\hline 45.62 & 50.21 \\
\hline 32.14 & 23.30 \\
\hline
\end{tabular}

JFig. 6] T2 MRI scan of a patient having anomaly in the right hemisphere 


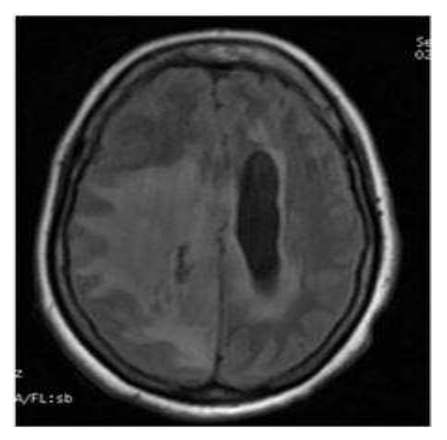

\begin{tabular}{|l|l|}
\hline 101.99 & 105.23 \\
\hline 116.12 & 118.02 \\
\hline 120.35 & 122.65 \\
\hline 131.25 & 134.25 \\
\hline
\end{tabular}

[Fig. 7] T2 MRI scan of a patient having tumor in the right hemisphere and its corresponding mean pixel values.

As from the above output, it is quite evident that high degree of symmetry does exist and can be very effectively represented with the help of mean pixel value parameter.

However in cases having anomalies, the mean pixel values of both hemispheres deviated and are different by large margin. These different values or difference can be very effectively used to detect anomaly is present or not. Same or identical mean pixel values gradient of both right and left hemisphere will illustrate no anomaly and different values will represent presence of any anomaly. The only reason for using T2 weighted MRI images because in T2-weighted images, tumors and edemas appear hyper intense.

The third phase consists of a case in which symmetry based approaches failed or unable to detect anomaly. One of the biggest drawback of all symmetry based approaches lies in their ability to detect anomaly present near the midline and on the midline in an axial MRI scan. In these cases entire robustness of the symmetry based approach to detect anomaly got compromised or failed. As anomaly detection by symmetry based approach is based on harnessing the intensity difference in between the right and left hemisphere. If the tumor is present on the midline or near the midline then the difference in intensity comes out to be zero as the half portion of tumor present in left hemisphere and another half in right hemisphere. So when the left and right modules pixel values got subtracted then the result always comes to be zero or very small highlighting no anomaly. Some of the typical cases in which tumor either present near the midline or on the midline presented below.

In the above case as the tumor is located just on the midline which separates the right and left hemisphere. In this case when we tend to apply the conventional symmetry based approach to detect the tumor by simply subtracting the left hemisphere from the right one in order to harness the difference in between the left and right hemisphere, the result always comes out to be zero or null. This happens because as the tumor is centrally located so half 
portion of it lies with the right hemisphere and exactly half portion with the left hemisphere which appears to be normal.

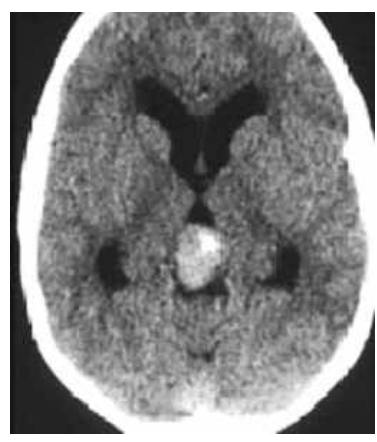

\begin{tabular}{|l|l|}
\hline 17.2034 & 17.1365 \\
\hline 41.60 & 42.03 \\
\hline 45.65 & 46.02 \\
\hline 24.3618 & 21.3 \\
\hline
\end{tabular}

[Fig. 8] MRI scans of a patient having tumor in the midline just in between the right and left hemisphere.

In these cases symmetry remains present and solely symmetry based approach cannot be used to detect the tumor in the above case. Apart from this other MRI examples of midline tumor are given below:
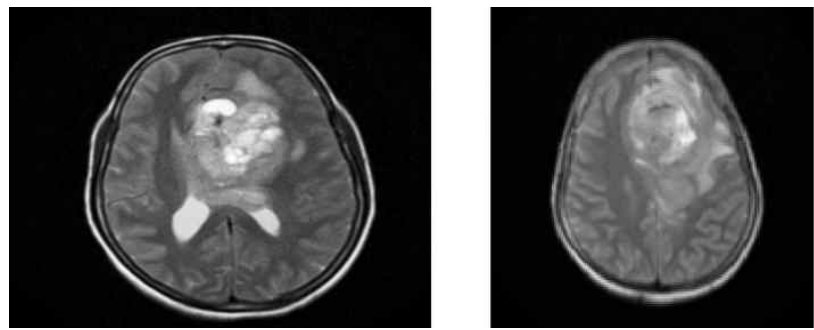

[Fig. 9] T2 MRI scans of a patient having large tumor in the midline.

\section{Conclusion}

All the symmetry based approaches are very popular because of its simple, effective and robust nature when it comes to detect anomaly in the MRI of a human brain. The new parameter i.e the mean pixel value gradient shows the high degree of symmetry is present in between the left and right hemisphere in normal human brain MRI and this symmetry is absent in abnormal human brain MRI. However it has been observed from our analysis that the symmetry property prove to be ineffective especially if the location of the tumor is near the Symmetry mid line or on the mid line. In such cases, the symmetry property alone cannot 
be used to detect tumor. It's a huge drawback present in all the approaches based on symmetry to detect the tumor. The main reason behind using T2-weighted image is the clear visibility of tumors in this type of MRI modality. In T2-weighted images, tumors and edemas appear hyper intense (brighter than the normal brain tissue) and hence easily detected.

\section{References}

[1] M.C Clark, Automatic tumor segmentation using knowledge-based techniques, IEEE Transactions Medical Imaging, (1998), Vol.17, No.2, pp.187-201.

[2] T. McInerney and D Terzopoulos, Deformable models in medical image analysis: a survey, Medical Image Analysis, (1996), Vol.1, No.2, pp.91-108.

[3] A Pitiot, Expert knowledge-guided segmentation system for brain MRI, Neuro image 23(Suppl. 1), (2004) pp.85-96.

[4] K. Doi, Current status and future potential of computer-aided diagnosis in medical imaging, Britain Institute of Radiology, (2005), 78:S3 - S19.

[5] M. A Huettig, Diagnostic expert system for structured reports, quality assessment, and training of residents in sonography, Med Klin (Munich), (2004), Vol.99, No.3, pp.117-22.

[6] S.V Thomas, An expert system for the diagnosis of epilepsy: results of a clinical trial, Natl Med J India, (2001), Vol.14, No.5, pp.274-276.

[7] S. Prima, S. Ourselin and N Ayache, Computation of the mid-sagittal plane in 3-D brain images, IEEE Transaction Medical imaging, (2002), Vol.21, No.2, pp.122-138.

[8] M. Frederik, V.L Koen, D.L Lynn, V Dirk and S Paul, Quantification of Cerebral Grey and White Matter Asymmetry from MRI, (1999), In MICCAI.

[9] J. F Iaccino, Left brain-right brain differences: inquiries evidence and new approaches. Hillsdale, NJ: Lawrence Erlbaum Associates, (1993).

[10] D. Blatter, A normative database from magnetic resonance imaging, In Neuroimaging I: basic science. Pletinum Press, (1996), pp.79-95.

[11] K Amunts, Interhemispheric asymmetry of human motor cortext related to handedness and gender, Neurospsychologia, (2000), 38:304 - 12.

[12] B. Weir, The history of cerebral vasospasm. Neurosurgery, Clin N Am, (1990), 1(1):265-76.

[13] V. Minoshima, A diagnostic approach in Alzheimer's disease using threedimensional stereotactic surface projections of fluorine-18-FDG PET, J Nucl Med, (1995), 36(7):1238 - 48.

[14] P Marias, R Guillernaud, M. Sakuma, A Zisserman and M Brady, Visualising cerebral asymmetry in visualization in biomedical computing, Springer, (1996), 411-16.

[15] J. M. Lee, J. J. Kim, I, Y, Kim, D. S. Lee, J. S. Kwon and S. I. Kim, Analysis of the hemispheric 
asymmetry using fractal dimension of a skeletonized cerebral surface, IEEE Transactions on Biomedical Engineering, (2004), 51(8):1494-8.

[16] V. A Kovalev, F Kruggel and D. Y von Cramon, Structural brain asymmetry as revealed by 3D texture analysis of anatomical MR images, In Proceedings of 16th international conference on pattern recognition, (2002).

[17] I. Volkau, Quantitative analysis of brain asymmetry by using the divergence measure: normal-pathological brain discrimination, Acad Radiol, (2006), 13:752 - 8 .

[18] J. P. Thirion, Statistical analysis of normal and abnormal dissymmetry in volumetric medical images. Medical Image Analysis, (2000), 4(2):111 - 21.

[19] L. P. Clarke, MRI: stability of three supervised segmentation techniques, Magnetic Resonance Imaging, (1993), 11(1):95 - 106.

[20] S. X. Liu, C. Imielinska, A. Laine, W. S Millar, E. S. Connolly and A. L. D'Ambrosio, Asymmetry analysis in rodent cerebral ischemia models, Academic Radiology, (2008), 15(9):1181 - 97.

[21] X. Liu, Statistical bilateral asymmetry measurement in brain images, In: IEEE 2006 international conference of the engineering in medicine and biology society, (2006), New York.

[22] J. R Atallah, On symmetry detection, IEEE Transaction on computers, (1985), pp.663-666.

[23] Y. Xia, Skeletonization via the realization of the fire front propagation and extinction in digital binary shapes, IEEE Transaction on pattern analysis and machine intelligence, (1989), pp.1076-1086.

[24] D Reisfeld, H. Wolfson and Y. Yeshurun, Detection of interest points using symmetry, Proceedings of ICCV, (1990), pp.62-65; Tokyo, Japan.

[25] P. Kovesi, Symmetry and Asymmetry from local phase, Proceedings of the 10th Australian Joint. Conference on Artificial Intelligence, (1997), pp.185-190.

[26] Y. Keller, An algebraic approach to symmetry detection, Proceedings of ICPR, (2004); Cambridge, U.K..

[27] A. V. Tuzikov, O. Colliot and I. Bloch, Evaluation of the symmetry plane in 3D MR brain images, Pattern Recognition Letters, (2003).

[28] Z. Wang, Q. Hu, K. Loe, A. Aziz and W. L Nowinski, Rapid and Automatic Detection of Brain Tumors in MR images, Proceedings of SPIE Medical Imaging, (2004); San Diego, USA.

[29] N. Ray, B. N. Saha, M. Brown and R. Graham, Locating Brain Tumors from MR Imagery Using Symmetry, Asilomar Conference on Signals, Systems, and Computers. Pacific Grove, (2007); California., USA.

[30] N. Ray, R. Greiner and A. Murtha, Using Symmetry to Detect Abnormities in Brain. MRI, Computer Society of India Communications, (2008), 31(19).

[31] S. Goyal, S. Shekhar and K. K Biswas, Automatic Detection of Brain Abnormalities, (2010), http://www.cse.iitd.ac.in/ cs5090255/autocom/paper.pdf. Accessed on 8th January 2017.

[32] P. Dvorak and W. Kropatsch, Detection of Brain Tumors Based on Automatic Symmetry Analysis, 18th Computer Vision Winter Workshop, (2013) February 4-6, 2013; Austria. 
[33] L. M. Shah and K. L. Salzman, Imaging of Spinal Metastatic Disease, International Journal of Surgical Oncology (2011). 